\title{
Relationship of cortisol, norepinephrine, and epinephrine levels with war-induced posttraumatic stress disorder in fathers and their offspring
}

\author{
Seyyed Taha Yahyavi, ${ }^{1,2}$ Mehran Zarghami, ${ }^{2,3}$ Farshad Naghshvar, ${ }^{4}$ Ahmad Danesh ${ }^{5}$ \\ ${ }^{1}$ Department of Psychiatry, School of Medicine, Roozbeh Hospital, Tehran University of Medical Sciences, Tehran, Iran. ${ }^{2}$ Psychiatry and \\ Behavioral Sciences Research Center, Addiction Institute, Mazandaran University of Medical Sciences, Sari, Mazandaran, Iran. ${ }^{3}$ Department \\ of Psychiatry, School of Medicine, Mazandaran University of Medical Sciences, Sari, Mazandaran, Iran. ${ }^{4}$ Department of Pathology, School of \\ Medicine, Mazandaran University of Medical Sciences, Sari, Mazandaran, Iran. ${ }^{5}$ School of Medicine, Golestan University of Medical Sciences, \\ Gorgan, Golestan, Iran.
}

\begin{abstract}
Objective: To compare afternoon serum/plasma levels of hormones in four groups: $(A)$ veterans with posttraumatic stress disorder (PTSD), (B) offspring of PTSD veterans, (C) veterans without PTSD, and (D) offspring of non-PTSD veterans.

Methods: Evaluation consisted of a semi-structured interview for axis I and II diagnoses, followed by measurement of afternoon serum cortisol and plasma epinephrine and norepinephrine by ELISA (Diametra) and LND (LDN Labor Diagnostika Nord GmbH \& Co. KG) respectively. Data were analyzed using descriptive statistics and the Student $t$, Kolmogorov-Smirnov, and nonparametric Mann-Whitney tests.

Results: One hundred and sixty-eight volunteers were investigated across the four groups. The groups were similar in terms of demographic characteristics, war experience and traumatization, and psychiatric and medical conditions other than PTSD (group A was similar to group C and group B was similar to group D). Between-groups comparisons did not yield statistically significant differences. Post-hoc analyses revealed significant differences in afternoon cortisol level between the offspring of veterans with current/past history of PTSD and the offspring of veterans without a history of PTSD. Conclusion: We only found decreased cortisol levels in offspring of veterans after rearranging the groups to reflect previous history of PTSD. Further studies are required to investigate the relationship between cortisol levels and the transgenerational effects of trauma and parental PTSD.
\end{abstract}

Keywords: Epinephrine; norepinephrine; cortisol; posttraumatic stress disorder; offspring

\section{Introduction}

Posttraumatic stress disorder (PTSD) is a psychopathological response to an extreme stressor. It is characterized by the presence of three symptom clusters, namely: intrusive re-experiencing of the trauma, avoidance of stimuli associated with the trauma, and increased physiological arousal with prominent anxiety and depressive features persisting for at least one month. ${ }^{1-3}$ In an updated World Health Organization (WHO) report published in 2009, using the Sheehan Disability Scale (SDS) to assess the Global Burden of Disease (GBD), the mean disability ratings for PTSD in developed and developing countries were 54.8 and $41.2 \%$ respectively. This indicates a high burden of PTSD, exceeding that of cancer (16.6 and $23.9 \%$ ), diabetes (13.6 and $23.7 \%$ ), and heart disease (26.5 and $25.7 \%){ }^{4}$ The burden of PTSD may be even higher in Iran, a country that was exposed to almost a decade of war: a 1991 study revealed a PTSD

Correspondence: Mehran Zarghami, Professor of Psychiatry, Zare Hospital, Taravat Street, Neka Road, Sari, Mazandaran, Iran.

E-mail: mzarghami@mazums.ac.ir

Submitted Mar 30 2014, accepted May 242014. prevalence of $29.1 \%$ among injured war veterans and $18.1 \%$ among soldiers involved in the Iran-Iraq war. ${ }^{5}$

There is inconsistent evidence that levels of cortisol, epinephrine, and norepinephrine in patients with PTSD and their offspring are different from those of the general population. Some studies show that PTSD is associated with decreased cortisol levels, ${ }^{6-9}$ while others indicate no change ${ }^{10-12}$ or even increased levels. ${ }^{13-18}$ There is also some limited evidence of decreased cortisol levels in the offspring of patients with PTSD. ${ }^{19-23}$ Some studies evaluating epinephrine and norepinephrine found increased levels even at rest, ${ }^{24}$ while others reported an exaggerated response only after trauma-related stimulation. ${ }^{25}$ Some authors believe that the correlation between the hypothalamic-pituitary-adrenal (HPA) axis and adrenergic system activity is negative in PTSD, as opposed to positive in depression. One study used norepinephrine/ cortisol (N/C) ratio to distinguish patients with PTSD from patients with other psychiatric disorders. ${ }^{26}$ Discrimination was achieved between PTSD and other diagnostic groups, indicating that the accuracy of the N/C ratio is substantially greater for the differential diagnosis of PTSD than the measurement of either of its two component hormones alone. ${ }^{27}$ 
Table 1 Demographic characteristics of veterans with and without PTSD and their offspring

\begin{tabular}{|c|c|c|c|c|}
\hline & $\begin{array}{l}\text { Combat veterans with } \\
\text { PTSD }(n=41)\end{array}$ & $\begin{array}{l}\text { Offspring of PTSD combat } \\
\text { veterans }(n=41)\end{array}$ & $\begin{array}{l}\text { Combat veterans without } \\
\text { PTSD }(n=43)\end{array}$ & $\begin{array}{l}\text { Offspring of non-PTSD } \\
\text { combat veterans }(n=43)\end{array}$ \\
\hline \multicolumn{5}{|l|}{ Sex } \\
\hline Male & $41(100)$ & $35(85)$ & $43(100)$ & $31(75)$ \\
\hline Female & $0(0)$ & $6(15)$ & $0(0)$ & $12(25)$ \\
\hline Total & 41 & 41 & 43 & 43 \\
\hline \multicolumn{5}{|l|}{ Age } \\
\hline Minimum & 40 & 8 & 41 & 10 \\
\hline Maximum & 64 & 27 & 57 & 29 \\
\hline Mean \pm SD & $47 \pm 5.7$ & $18 \pm 4.4$ & $48 \pm 4.4$ & $19 \pm 4.7$ \\
\hline \multicolumn{5}{|l|}{ Education } \\
\hline Illiterate & $2(5)$ & $0(0)$ & $0(0)$ & $1(2)$ \\
\hline Primary & 9 (22) & 17 (42) & $10(23)$ & $19(44)$ \\
\hline Secondary & $23(56)$ & $23(56)$ & 21 (49) & $19(44)$ \\
\hline Bachelor's degree & $6(15)$ & $1(2)$ & $7(16)$ & $3(6)$ \\
\hline Master's degree & $1(2)$ & $0(0)$ & $5(12)$ & $2(4)$ \\
\hline Mean height $(\mathrm{cm})$ & 170 & 165 & 172 & 169 \\
\hline Mean weight $(\mathrm{kg})$ & 74 & 61 & 80 & 70 \\
\hline
\end{tabular}

Data expressed as absolute numbers (percentages), unless otherwise specified.

PTSD = posttraumatic stress disorder; SD = standard deviation.

Because further understanding of PTSD neuroendocrinology may contribute to patient management, we conducted a historical cohort study to examine the relationship between cortisol, epinephrine, and norepinephrine levels in combat veterans with PTSD and their offspring in Iran.

\section{Methods}

\section{Setting}

The study was carried out from September to November 2011. Veterans of the Iraq-Iran war living in the city of Sari, Iran, were invited to participate via short message service (SMS).

\section{Participants}

Eighty-four veterans, each with one child, volunteered to be included in the study. Semi-structured interviews for axis I and II diagnoses (SCID-I and SCID-II) ${ }^{28,29}$ were conducted. Based on the presence of full DSM-IV-TR criteria for PTSD in veterans, volunteers were divided into four groups: (A) 41 veterans with PTSD, (B) 41 offspring of PTSD veterans, $(C) 43$ veterans without PTSD, and (D) 43 offspring of non-PTSD veterans. The severity of PTSD in group $(A)$ was evaluated with the Mississippi Scale. ${ }^{30}$

\section{Laboratory measures}

Blood samples were obtained from participants in the afternoon (around 4p.m), at rest, and were frozen. Serum cortisol levels and simultaneous plasma epinephrine and norepinephrine levels were assessed with a commercially available ELISA kit (Diametra, Foligno, Italy) and LND (LDN Labor Diagnostika Nord GmbH \& Co. KG, Nordhorn, Germany), respectively.

\section{Statistical analysis}

Data were analyzed by means of descriptive statistics and the Student $t$, Kolmogorov-Smirnov, and nonparametric Mann-Whitney tests. All tests were carried out in SPSS version 17

\section{Ethical aspects}

All subjects volunteered to participate in the study and provided verbal or written informed consent. One case in group D refused to give a blood sample; hence, only 42 samples were obtained from this group. This study was approved by the Mazandaran University of Medical Sciences Research Ethics Committee.

\section{Results}

One hundred and sixty-eight subjects participated, and 167 blood samples were obtained. The demographic profile of the sample is displayed in Table 1.

\section{PTSD severity}

In group A (veterans with PTSD), 25.5\% of participants had mild PTSD (Mississippi Scale Score < 60), 54.5\% had moderate PTSD (scores between 60 and 120), and $20 \%$ had severe PTSD (score $>120$ ). The mean Mississippi Scale Score was 104.34 \pm 22.26 (minimum = 50 , maximum $=142$, median $=109.5$ )

\section{Veteran group characteristics}

Participants in groups $\mathrm{A}$ and $\mathrm{C}$ had been exposed to war during adolescence and early adulthood and were moderately to severely traumatized. Details are displayed in Table 2. 
Table 2 Comparison between the traumatic experiences of veterans in the PTSD and non-PTSD groups

\begin{tabular}{lcc}
\hline & $\begin{array}{c}\text { Combat veterans with current } \\
\text { PTSD (group A) }\end{array}$ & $\begin{array}{c}\text { Combat veterans without current } \\
\text { PTSD (group C) }\end{array}$ \\
\hline Mean age at first exposure to war (years) & $21.68 \pm 6.12$ & $20.61 \pm 4.69$ \\
Mean duration of exposure to war (months) & $22.34 \pm 14.22$ & $20.75 \pm 16.01$ \\
Number of veterans imprisoned and tortured in Iraq, n (\%) & $9(22)$ & $12(28)$ \\
Duration of war imprisonment (months) & $20.55 \pm 7.43$ & $29.90 \pm 15.47$ \\
Level of disability* & $41.19 \pm 12.33$ & $33.79 \pm 12.12$ \\
\hline
\end{tabular}

Data expressed as mean \pm standard deviation, unless otherwise specified.

PTSD = posttraumatic stress disorder.

* In Veterans' Affairs language and policy, level of disability refers to the degree of different organ's dysfunction, which may be partial or total.

The conditions of injured veterans' disability will influence how their injury is categorized by the Veterans' Affairs medical commission.

\section{Offspring group characteristics}

The mean time (years) between paternal exposure to trauma and offspring birth was 8.4 years in group $B$ and 9.3 years in group $D$, which was not significantly different.

\section{Axis I and III conditions in participants}

The distribution of axis I and III conditions, as evaluated by SCID-I and an unstructured interview respectively, is displayed in Table 3. No significant difference in psychiatric and medical conditions was observed across the groups, except for PTSD. Thirteen veterans in group $C$ had a past psychiatric history of combat-related PTSD that was in remission at the time of interview.

\section{Comparisons between groups}

Since the catecholamine and cortisol levels were not normally distributed, these variables were compared between groups (A vs. C and B vs. D) with the nonparametric Mann-Whitney $U$ test. No significant differences were observed between groups. Because the comparison between veterans and their offspring would involve several confounding factors, such as age and life experiences, we did not try to compare all four groups to one another.

\section{Post-hoc analyses}

In a post-hoc analysis, after omission of 13 non-PTSD veterans with past psychiatric history of combat related PTSD (as well as their offspring), a significant difference in cortisol levels was found between the offspring of PTSD and non-PTSD veterans $(p=0.02)$. In addition, by adding these 13 non-PTSD veterans with past psychiatric history of PTSD to group A and their offspring to group B, a significant difference in cortisol level was observed between offspring of veterans with history of PTSD (group B) and offspring of veterans without history of PTSD (group D) $(p=0.045)$. The details are illustrated in Tables 4 and 5.

\section{Discussion}

Of the 168 volunteers who participated in the study, 167 consented to blood sampling. Participants in both veteran

Table 3 Present axis I and III diagnoses (relevant medical conditions) in veterans with and without PTSD and their offspring

\begin{tabular}{|c|c|c|c|c|}
\hline & $\begin{array}{l}\text { Combat veterans with } \\
\text { PTSD }(n=41)\end{array}$ & $\begin{array}{l}\text { Offspring of PTSD combat } \\
\text { veterans }(n=41)\end{array}$ & $\begin{array}{c}\text { Combat veterans without } \\
\text { PTSD }(n=43)\end{array}$ & $\begin{array}{l}\text { Offspring of non-PTSD } \\
\text { combat veterans }(n=43)\end{array}$ \\
\hline PTSD & $41(100.0)$ & 0 & 0 & $1(2.3)$ \\
\hline MDD & $5(12.2)$ & $1(2.4)$ & $3(7.0)$ & 0 \\
\hline GAD & 0 & $4(9.7)$ & 0 & $2(4.7)$ \\
\hline Schizophrenia spectrum & $1(2.4)$ & 0 & 0 & $1(2.3)$ \\
\hline OCD & $2(4.9)$ & $3(7.3)$ & $1(2.3)$ & $6(14.3)$ \\
\hline Phobia & $1(2.4)$ & $12(29.2)$ & $4(9.3)$ & $13(31.0)$ \\
\hline Panic disorder & $1(2.4)$ & 0 & $2(4.7)$ & 0 \\
\hline Anxiety disorder NOS & 0 & $1(2.4)$ & $3(7.0)$ & $1(2.3)$ \\
\hline Impulse control disorder & $2(4.9)$ & 0 & $2(4.7)$ & 0 \\
\hline Somatoform disorder & $4(9.7)$ & $3(7.3)$ & $2(4.7)$ & 0 \\
\hline Primary sleep disorder & $2(4.9)$ & 0 & $3(7.0)$ & $2(4.7)$ \\
\hline ADHD & 0 & $2(4.9)$ & 0 & $2(4.7)$ \\
\hline Tic disorder & 0 & $1(2.4)$ & 0 & $1(2.3)$ \\
\hline Adjustment disorder & 0 & 0 & $1(2.3)$ & 0 \\
\hline \multicolumn{5}{|l|}{ Medical disease } \\
\hline None & 1 & 37 & 11 & 39 \\
\hline Mild & 22 & 4 & 20 & 1 \\
\hline Moderate & 12 & 0 & 7 & 2 \\
\hline Severe & 6 & 0 & 5 & 1 \\
\hline
\end{tabular}

Data expressed as absolute numbers (percentages).

$\mathrm{ADHD}=$ attention-deficit/hyperactivity disorder; $\mathrm{GAD}=$ generalized anxiety disorder; $\mathrm{MDD}=$ major depressive disorder; NOS = not otherwise specified; OCD = obsessive-compulsive disorder; PTSD = posttraumatic stress disorder. 
Table 4 Cortisol, epinephrine, and norepinephrine levels and epinephrine/cortisol and norepinephrine/cortisol ratios in veterans with and without a history of PTSD

\begin{tabular}{lccc}
\hline & Veterans with history of & Veterans without history of \\
& PTSD $(\mathrm{n}=54)$ & PTSD $(\mathrm{n}=30)$ & $\mathrm{p}$-value \\
\hline Epinephrine $(\mathrm{pg} / \mathrm{mL})$ & $51.10 \pm 6.49$ & $43.04 \pm 6.83$ & 0.33 \\
Norepinephrine $(\mathrm{pg} / \mathrm{mL})$ & $359.03 \pm 46.59$ & $311.33 \pm 29.40$ & 0.97 \\
Cortisol $(\mathrm{ng} / \mathrm{mL})$ & $144.33 \pm 9.31$ & $141.12 \pm 9.90$ & 0.76 \\
Epinephrine to cortisol ratio & $0.35 \pm 0.07$ & $0.30 \pm 0.06$ & 0.66 \\
Norepinephrine to cortisol ratio & $2.48 \pm 0.53$ & $2.20 \pm 0.53$ & 0.62 \\
\hline
\end{tabular}

Data expressed as mean \pm standard deviation.

PTSD = posttraumatic stress disorder; SD =standard deviation.

groups ( $\mathrm{A}$ and $\mathrm{C}$ ) were middle-aged and had been exposed to war trauma in early adulthood. There were no significant differences in the duration and severity of traumatization, education, height, weight, medical problems, or psychiatric comorbidities. The severity of PTSD in group A was moderate, based on the self-report Mississippi Scale for Combat-Related PTSD Severity. Participants in both offspring groups ( $B$ and $D)$ were, on average, in adolescence. There were no significant differences between the two groups in height, weight, medical problems, psychiatric comorbidities, or interval between paternal traumatization and offspring birth. In post-hoc analyses, there was a significant difference in afternoon serum cortisol levels between offspring of veterans with a current or past history of combat-related PTSD and offspring of veterans who did not have any history of PTSD. Our findings are consistent with studies reporting lower serum or urinary cortisol levels in offspring of patients with PTSD compared with offspring of nonPTSD patients, ${ }^{19,21,22,31}$ and normal epinephrine and norepinephrine levels at rest. 25,32

As mentioned earlier, there is a wide range of evidence about the hormonal and neurobiological features of PTSD; in some aspects, these findings might be paradoxical. Yehuda et al. conducted a series of studies on small samples of Holocaust survivors and their offspring. They found lower cortisol levels in both PTSD survivors and the next generation. 6,7,19,20,22,23,30,31,33-36 There are also several studies that show normal and even increased cortisol levels in patients with PTSD. ${ }^{10,11,15,16,18}$

Yehuda et al. also conducted research on pregnant mothers exposed to the September 11, 2001 attack and observed significantly lower levels of cortisol among newborns of mothers with PTSD than among those born to non-PTSD mothers. ${ }^{21}$ In our review of the MEDLINEindexed literature, we did not find any research on the alteration of newborn cortisol levels exclusively in the context of offspring of trauma-exposed fathers. However, paternal transmission of pathological predisposition may differ in characteristics and pathways from its maternal counterpart.

There are few studies about the cortisol levels of offspring of PTSD patients in the medical literature. This might explain the inconsistent evidence about alterations in cortisol levels in PTSD patients, or could be related to lesser interest of journals in publishing non-significant results. Yehuda et al. mainly studied a specific population exposed to specific, severe trauma. To generalize their findings to other populations, further studies with larger samples, different populations, and diverse types and severities of trauma might be essential. Furthermore, it should be emphasized that severe trauma exposure is not necessarily synonymous with PTSD diagnosis. Yehuda et al. diagnosed PTSD retrospectively in the context of psychological autopsy in their Holocaust studies. In contrast, the non-PTSD veterans (control group) in our sample had all experienced severe war trauma, with traumatization that was similar in duration and severity to that experienced by PTSD patients.

In the present study, we did not find any significant differences in cortisol level between the PTSD and nonPTSD groups ( $A$ and $C$ ). This could be the effect of confounding factors such as time point of cortisol assessment or other mood-anxiety comorbidities. Despite this result, specific attention should be paid to the inconsistent evidence about PTSD and cortisol levels in the literature. A review by Meewisse et al. about cortisol levels in PTSD postulated that several factors, including the type and severity of trauma, age of exposure, gender,

Table 5 Cortisol, epinephrine, and norepinephrine levels and epinephrine/cortisol and norepinephrine/cortisol ratios in offspring of veterans with and without a history of PTSD

\begin{tabular}{lccc}
\hline & $\begin{array}{c}\text { Offspring of veterans with } \\
\text { history of PTSD }(\mathrm{n}=54)\end{array}$ & $\begin{array}{c}\text { Offspring of veterans without } \\
\text { history of PTSD }(\mathrm{n}=30)\end{array}$ & $\mathrm{p}$-value \\
\hline Epinephrine $(\mathrm{pg} / \mathrm{mL})$ & $47.91 \pm 6.60$ & $46.02 \pm 6.33$ & 0.73 \\
Norepinephrine $(\mathrm{pg} / \mathrm{mL})$ & $355.80 \pm 35.90$ & $375.44 \pm 45.48$ & 0.76 \\
Cortisol $(\mathrm{ng} / \mathrm{mL})$ & $121.30 \pm 8.04$ & $142.05 \pm 6.81$ & $0.045^{*}$ \\
Epinephrine to cortisol ratio & $0.39 \pm 0.06$ & $0.32 \pm 0.05$ & 0.23 \\
Norepinephrine to cortisol ratio & $2.89 \pm 0.40$ & $2.64 \pm 0.39$ & 0.25 \\
\hline
\end{tabular}

Data expressed as mean \pm standard deviation.

PTSD = posttraumatic stress disorder; SD = standard deviation

* Statistically significant. 
genetics, chronicity, and severity of PTSD symptoms, would have an essential role in increasing or decreasing the cortisol level. ${ }^{37}$

There are two major limitations in our study. The first is the small sample size, which resulted in the non-normal distribution of catecholamine and cortisol levels. Second, we measured cortisol levels, which vary with the circadian rhythm, in spot blood samples. Although we planned and conducted our study on the basis of the Meewisse et al. review, which postulated that PTSD patients would have their nadir cortisol levels in the afternoon, ${ }^{37}$ 24-hour urinary cortisol may be a more reliable index. Furthermore, it seems that measurement of epinephrine and norepinephrine after trauma-like stimulation may be associated with a more exaggerated response in PTSD patients; hence, a significant difference from the normal population may result. ${ }^{25}$

The cortisol and catecholamine systems are highly sensitive. Therefore, excluding all axis I, II, and III disorders would result in a sample of homogeneous patients with a possible unique hormonal profile. However, a study on such a homogeneous group of patients would not be externally valid. In other words, considering that comorbidity is a rule rather than an exception in PTSD, ${ }^{38}$ the congruency of possible findings from these "pure PTSD" patients with the majority of "clinical" PTSD patients should be investigated.

Finally, we must stress the complex nature of the cortisol and catecholamine systems, which make prescribing a simple formulation impossible. Future studies should focus on hormonal profile, preferably measuring 24-hour cortisol and post-stimulation catecholamine levels, in larger, homogeneous samples. This would enable comparison with a non-traumatized normal control group.

\section{Acknowledgements}

This study was supported by a grant from the Mazandaran University of Medical Sciences. It is part of the first author's postgraduate thesis. The authors express their deep gratitude to the Department of Veterans' Affairs-Mazandaran Branch, especially the research branch directed by Dr. Taghavi, the health branch directed by Dr. Mirsadeghi, and all other staff, including Mr. Damsi, for their cooperation. We also thank the staff at the Zare hospital, especially the manager, department of psychotherapy, laboratory, and library. We also appreciate the collaboration of the Sari Central Pathobiology laboratory staff and management, especially Mrs. Colbadi.

\section{Disclosure}

The authors report no conflicts of interest.

\section{References}

1 American Psychiatric Association. Diagnostic and Statistical Manual of Mental Disorders, Fourth Edition, Text Revision (DSM-IV-TR). Arlington: American Psychiatric Publishing; 2000.
2 Sanati M, Zarghami M, Keshoufi M. [Anxiety features in PTSD veterans]. J Facul Med. 1991;49:43-55.

3 Sanati M, Zarghami M. Kashoufi M. [Depression in chronic combat reaction]. J Facul Med. 1995;53:75-8.

4 Kessler RC, Aguilar-Gaxiola S, Alonso J, Chatterji S, Lee S, Ormel J, et al. The global burden of mental disorders: an update from the WHO World Mental Health (WMH) surveys. Epidemiol Psichiatr Soc. 2009;18:23-33.

5 Norbala A, Malekafzali $\mathrm{H}$. Review dimensions war effects on four groups of different people of Tehran. In: 3th Congress of Medical Psychology Research; 1992; Tehran, Iran.

6 Yehuda R, Southwick SM, Nussbaum G, Wahby V, Giller EL Jr, Mason JW. Low urinary cortisol excretion in patients with posttraumatic stress disorder. J Nerv Ment Dis. 1990;178:366-9.

7 Yehuda R, Kahana B, Binder-Brynes K, Southwick SM, Mason JW, Giller EL. Low urinary cortisol excretion in Holocaust survivors with posttraumatic stress disorder. Am J Psychiatry. 1995;152:982-6.

8 Rohleder N, Joksimovic L, Wolf JM, Kirschbaum C. Hypocortisolism and increased glucocorticoid sensitivity of pro-Inflammatory cytokine production in Bosnian war refugees with posttraumatic stress disorder. Biol Psychiatry. 2004;55:745-51.

9 Thaller V, Vrkljan M, Hotujac L, Thakore J. The potential role of hypocortisolism in the pathophysiology of PTSD and psoriasis. Coll Antropol. 1999;23:611-9.

10 Sondergaard HP, Theorell T. A longitudinal study of hormonal reactions accompanying life events in recently resettled refugees. Psychother Psychosom. 2003;72:49-58.

11 Mason JW, Giller EL, Kosten TR, Ostroff RB, Podd L. Urinary freecortisol levels in posttraumatic stress disorder patients. J Nerv Ment Dis. 1986;174:145-9.

12 De Bellis MD, Baum AS, Birmaher B, Keshavan MS, Eccard CH, Boring AM, et al. A.E. Bennett Research Award. Developmental traumatology. Part I: Biological stress systems. Biol Psychiatry. 1999;45:1259-70

13 Song $\mathrm{Y}$, Zhou D, Wang X. Increased serum cortisol and growth hormone levels in earthquake survivors with PTSD or subclinical PTSD. Psychoneuroendocrinology. 2008;33:1155-9.

14 Rasmusson AM, Lipschitz DS, Wang S, Hu S, Vojvoda D, Bremner $\mathrm{JD}$, et al. Increased pituitary and adrenal reactivity in premenopausal women with posttraumatic stress disorder. Biol Psychiatry. 2001; 50:965-77.

15 Pitman RK, Orr SP. Twenty-four hour urinary cortisol and catecholamine excretion in combat-related posttraumatic stress disorder. Biol Psychiatry. 1990;27:245-7.

16 Liberzon I, Abelson JL, Flagel SB, Raz J, Young EA. Neuroendocrine and psychophysiologic responses in PTSD: a symptom provocation study. Neuropsychopharmacology. 1999;21: 40-50.

17 Lemieux AM, Coe CL. Abuse-related posttraumatic stress disorder: evidence for chronic neuroendocrine activation in women. Psychosom Med. 1995;57:105-15.

18 Maes M, Lin A, Bonaccorso S, van Hunsel F, Van Gastel A, Delmeire $\mathrm{L}$, et al. Increased 24-hour urinary cortisol excretion in patients with post-traumatic stress disorder and patients with major depression, but not in patients with fibromyalgia. Acta Psychiatr Scand. 1998;98:328-35.

19 Yehuda R, Bierer LM, Schmeidler J, Aferiat DH, Breslau I, Dolan S. Low cortisol and risk for PTSD in adult offspring of holocaust survivors. Am J Psychiatry. 2000;157:1252-9.

20 Yehuda R, Blair W, Labinsky E, Bierer LM. Effects of parental PTSD on the cortisol response to dexamethasone administration in their adult offspring. Am J Psychiatry. 2007;164:163-6.

21 Yehuda R, Engel SM, Brand SR, Seckl J, Marcus SM, Berkowitz GS. Transgenerational effects of posttraumatic stress disorder in babies of mothers exposed to the World Trade Center attacks during pregnancy. J Clin Endocrinol Metab. 2005;90:4115-8.

22 Yehuda R, Halligan SL, Bierer LM. Cortisol levels in adult offspring of Holocaust survivors: relation to PTSD symptom severity in the parent and child. Psychoneuroendocrinology. 2002;27:171-80.

23 Yehuda R, Teicher MH, Seckl JR, Grossman RA, Morris A, Bierer LM. Parental posttraumatic stress disorder as a vulnerability factor for low cortisol trait in offspring of holocaust survivors. Arch Gen Psychiatry. 2007;64:1040-8. 
24 Kosten TR, Mason JW, Giller EL, Ostroff RB, Harkness L. Sustained urinary norepinephrine and epinephrine elevation in post-traumatic stress disorder. Psychoneuroendocrinology. 1987;12:13-20.

25 McFall ME, Veith RC, Murburg MM. Basal sympathoadrenal function in posttraumatic distress disorder. Biol Psychiatry. 1992; 31:1050-6.

26 Giller EL PB, Southwick S, Yehuda R, Wahby V, Kosten TR, et al Psychoendocrinology of posttraumatic stress disorder. In: Wolf ME, Mosnaim $A D$, editors. Posttraumatic stress disorder: etiology, phenomenology, and treatment. Washington: American Psychiatric Press; 1990. p. 158-67.

27 Mason JW, Giller EL, Kosten TR, Harkness L. Elevation of urinary norepinephrine/cortisol ratio in posttraumatic stress disorder. J Nerv Ment Dis. 1988;176:498-502.

28 Sharifi V, Assadi SM, Mohammadi MR, Amini H, Kaviani H, Semnani $\mathrm{Y}$, et al. Structured Clinical Interview for DSM-IV (SCID): Persian translation and cultural adaptation. Iran J Psychiatry. 2007;2:46-8.

29 Sharifi V, Assadi SM, Mohammadi MR, Amini H, Kaviani H, Semnani $Y$, et al. A Persian translation of the Structured Clinical Interview for Diagnostic and Statistical Manual of Mental Disorders, Fourth Edition: psychometric properties. Compr Psychiatry. 2009; 50:86-91.
30 Yehuda R, Halligan SL, Grossman R. Childhood trauma and risk for PTSD: relationship to intergenerational effects of trauma, parental PTSD, and cortisol excretion. Dev Psychopathol. 2001;13:733-53.

31 Yehuda R. Biology of posttraumatic stress disorder. J Clin Psychiatry. 2001;62:41-6.

32 McFall ME, Murburg MM, Ko GN, Veith RC. Autonomic responses to stress in Vietnam combat veterans with posttraumatic stress disorder. Biol Psychiatry. 1990;27:1165-75.

33 Yehuda R. Clinical relevance of biologic findings in PTSD. Psychiatr Q. 2002;73:123-33.

34 Yehuda R. Current status of cortisol findings in post-traumatic stress disorder. Psychiatr Clin North Am. 2002;25:341-68, vii.

35 Yehuda R, Bell A, Bierer LM, Schmeidler J. Maternal, not paternal, PTSD is related to increased risk for PTSD in offspring of Holocaust survivors. J Psychiatr Res. 2008;42:1104-11.

36 Yehuda R, Bierer LM. The relevance of epigenetics to PTSD: implications for the DSM-V. J Trauma Stress. 2009;22:427-34.

37 Meewisse ML, Reitsma JB, de Vries GJ, Gersons BP, Olff M. Cortisol and post-traumatic stress disorder in adults: systematic review and meta-analysis. Br J Psychiatry. 2007;191:387-92.

38 Sadock BJ, Sadock VA. Kaplan and Sadock's comprehensive textbook of psychiatry. Philadelphia: Lippincott Williams \& Wilkins; 2009. 